\title{
Impacto da Precisão Reduzida e Mista na Computação do Método de Lattice-Boltzmann em Múltiplas GPUs*
}

\author{
Gabriel Freytag ${ }^{1}$, João V. F. Lima ${ }^{2}$, Paolo Rech $^{1}$, Philippe O. A. Navaux ${ }^{1}$ \\ ${ }^{1}$ Universidade Federal do Rio Grande do Sul (UFRGS) - Porto Alegre - RS - Brasil \\ ${ }^{2}$ Universidade Federal de Santa Maria (UFSM) - Santa Maria - RS - Brasil \\ \{gfreytag,prech, navaux\}@inf.ufrgs.br, jvlima@inf.ufsm.br
}

\begin{abstract}
Resumo. A heterogeneidade de arquiteturas de computação modernas permite que engenheiros refinem algoritmos para maximizar o nível de afinidade com a arquitetura e, consequentemente, a eficácia da computação. Neste trabalho, investigamos o impacto da precisão reduzida e mista na computação do método de Lattice-Boltzmann em uma plataforma multi-GPU. Usando meia precisão para o armazenamento e simples para as operações aritméticas, obteve-se um speedup de 3.44 consumindo $71 \%$ menos energia e uma perda $0.02 \%$ de acurácia.
\end{abstract}

\section{Introdução}

Os Sistemas de Computação de Alto Desempenho (HPC) atualmente conectam um grande número de dispositivos com arquiteturas distintas que, por si só, estão tornando-se heterogêneas. No entanto, ao mesmo tempo em que a heterogeneidade dos sistemas aumenta para suportar diferentes características computacionais, a demanda por recursos computacionais excede a disponibilidade atual. Embora alguns problemas exijam resultados exatos, outros admitem resultados aproximados, reduzindo os requisitos de recursos e, consequentemente, melhorando o desempenho. Uma das estratégias mais prevalentes é o dimensionamento de precisão [Mittal 2016], que explora a precisão mista. Assim, é possível compensar a qualidade do resultado com desempenho e eficiência energética.

Neste artigo, avaliamos o impacto da precisão reduzida e mista no desempenho, eficiência energética e precisão da computação estêncil simultaneamente em 4 GPUs NVIDIA P100 com suporte nativo a precisão mista. Como estudo de caso, usamos a amplamente conhecida aplicação de Dinâmica de Fluídos Computacional (CFD) denominada método de Lattice-Boltzmann (LBM) com um modelo tridimensional D3Q19.

\section{Implementações}

Neste trabalho, foram implementadas três versões distintas do LBM. Na primeira, multigpu, as operações aritméticas de ponto flutuante e o armazenamento dos dados na memória utilizam a mesma precisão, selecionada em tempo de compilação. Esta versão executa todas as operações aritméticas com funções matemáticas regulares do CUDA. Já a segunda implementação, multigpu_float, executa todas as operações aritméticas de ponto flutuante com precisão simples, usando funções matemáticas intrínsecas do CUDA, e a precisão do armazenamento dos dados é definida em tempo de compilação em dupla, simples ou meia. Por fim, a terceira implementação, multigpu_half2, realiza duas

*Apoio financeiro CAPES. 
Tabela 1. Resultados experimentais em um modelo 3D de tamanho $352 \times 352 \times 352$.

\begin{tabular}{cccccc}
\hline Implementação & FP & Execução (s) & Speedup & Erro FP (\%) & Energia (kWh) \\
\hline \multirow{2}{*}{ multigpu } & double & 19.47 & 1.00 & 0.00 & 0.3919 \\
\cline { 2 - 6 } & single & 11.16 & 1.75 & 0.00 & 0.1418 \\
\cline { 2 - 6 } & half & 7.67 & 2.54 & 0.22 & 0.0648 \\
\hline \multirow{3}{*}{ multigpu_float } & double & 16.81 & 1.16 & 0.00 & 0.2652 \\
\cline { 2 - 6 } & single & 8.52 & 2.28 & 0.00 & 0.0734 \\
\cline { 2 - 6 } & half & 5.66 & 3.44 & 0.02 & 0.0326 \\
\hline \multirow{3}{*}{ multigpu_half2 2} & double & 17.37 & 1.12 & 0.06 & 0.2789 \\
\cline { 2 - 6 } & single & 8.72 & 2.23 & 0.06 & 0.0763 \\
\cline { 2 - 6 } & half & 5.85 & 3.33 & 0.06 & 0.0354 \\
\hline
\end{tabular}

operações aritméticas de meia precisão em paralelo em uma única Unidade de Ponto Flutuante (FPU) de precisão simples utilizando um tipo de dado vetorizado (half2) e funções matemáticas intrínsecas do CUDA, ao invés das funções matemáticas regulares.

\section{Resultados}

Na Tabela 1, é possível observar o tempo de execução, o speedup (com base na versão multigpu utilizando precisão dupla), a perda de precisão e o consumo de energia das três implementações em uma plataforma com 4 GPUs NVIDIA Tesla P100. A medida que reduzimos a precisão das operações aritméticas, o tempo de execução e o consumo de energia reduzem significativamente. Com meia precisão ao invés de precisão dupla, há um speedup de 2.54 e uma redução no consumo de energia de $58 \%$ com a mesma precisão para o armazenamento dos dados (multigpu). Já ao fixar a precisão das operações aritméticas em simples e utilizar funções matemáticas intrínsecas ao invés de funções regulares, é possível obter um speedup de 3.44 e reduzir o consumo de energia em $71.4 \%$ usando meia precisão para o armazenamento dos dados (multigpu_float). Por fim, ao fixar a precisão das operações aritméticas em meia, utilizando dados vetorizados (half2) e funções matemáticas intrínsecas (multigpu_half2), o desempenho se manteve significativamente próximo à implementação multigpu_float devido à falta de afinidade dos procedimentos do método com operações vetoriais, necessitando reestruturação total das mesmas.

\section{Conclusão e Trabalhos Futuros}

No presente trabalho, mostramos que é possível obter ganhos significativos de desempenho e consumo de energia ao fixar a precisão das operações aritméticas em simples e reduzir a precisão do armazenamento em meia precisão. Como trabalhos futuros, sugerimos a otimização da versão multigpu_half2 e a análise de outras aplicações de física.

\section{Referências}

Mittal, S. (2016). A survey of techniques for approximate computing. ACM Computing Surveys, 48(4). 